\title{
La cartografía bibliográfica y la memoria emblemática Playa Ancha, Valparaíso
}

\author{
Marjorie Mardones Leiva \\ Javiera Carmona Jiménez \\ Universidad de Playa Ancha - UPLA, Chile
}

ARTICLE

\begin{abstract}
Resumen
Este estudio propone un análisis para el estudio de la memoria emblemática del territorio de Playa Ancha, Valparaíso, basándose en una revisión bibliográfica situada. Su objetivo principal es reconocer los discursos que han colaborado en la construcción, articulación y cristalización del imaginario social de la localidad a partir de su producción bibliográfica: temáticas recurrentes, agentes discursivos oficiales y disidentes, prácticas sociales, entre otros. Esta investigación pone en crisis la tendencia de los estudios bibliométricos actuales que se han dedicado principalmente al análisis de información para entidades educacionales o de producción científica nacional, dejando atrás aspectos tan importantes como es el espacio, tiempo, lugar (Sousa-Santos, 1991) y su relación con el día a día del ciudadano. Para representar los resultados del estudio, se han utilizado gráficos y cartografías, destacando la relación entre territorio y bibliografía.
\end{abstract}

Palabras clave

Cartografía bibliográfica ; Bibliometría ; Territorio ; Memoria emblemática ; Memoria bibliográfica

\section{Mapping the literature and memory flagship Playa Ancha, Valparaíso}

\begin{abstract}
This study proposes an analysis for the study of the emblematic memory of the territory of Playa Ancha, Valparaiso, based on a located bibliographic review. Its main aim is to recognize the discourses that collaborated in the construction, articulation and crystallization of local social imaginary from its bibliographic production: recurrent themes, official discursive agents and dissidents, social practices, among others. This research puts in crisis the tendency of current bibliometric studies that had been dedicated mainly to the analysis of information for educational institutions or national scientific production, leaving behind such important aspects as space, time, place (Sousa-Santos, 1991) And their relationship with the day-to-day of the citizen. For to represent the results of the study, were used graphs and cartographics, highlighting the relationship between territory and bibliography.
\end{abstract}

\section{Keywords}

Bibliographic cartography ; Bibliometrics ; Mapping ; Territory ; Flagship memory ; Bibliographic memory

\section{Introducción}

El estudio de la memoria ha cobrado importancia creciente en el ámbito de las ciencias sociales en las últimas décadas, referidos en particular a la tensión entre una memoria emblemática y las memorias sueltas y fragmentadas producto de los traumas sociales que afectan de manera desigual la estructura social. Tal ha sido el caso de las dictaduras, catástrofes (terremotos, maremotos, erupciones volcánicas) y eventos (incendios, explosiones, crímenes) que generan "memorias difíciles" vinculadas con un "patrimonio abyecto" en la construcción de la identidad nacional (Garretón, 2003). El estudio de las identidades locales o territoriales se ha centrado en la fragmentación de las grandes ciudades (Márquez, 2008) y sobre sectores periféricos en el marco de la discusión teórica sobre el desarrollo local (Di Pietro, 2001). Es así como la aproximación a la identidad, a la discusión sobre la modernidad "inacabada" y la construcción de sujetos colectivos tienen en los estudios sobre la memoria los conceptos y procesos fundantes. 
Es en la disputa sobre la simbolización del poder donde la memoria activa procesos de patrimonialización dirigidos. En tales procesos el patrimonio adquiere formas intangibles (no por ello inaprehensibles) bajo la denominación de "bien cultural", noción que expresa una operación de inclusión y a la vez de exclusión de elementos sobre un repertorio en construcción permanente (Ariño, 2002). La noción de "bien cultural" también revela la pluralidad de agentes que lo activan, tales como el Estado, expertos "voceros" de las universidades y fuerzas armadas, organismos internacionales, organizaciones sociales, etc. El incremento de los patrimonios potenciales se unen inextricablemente a los agentes que intervienen en su patrimonialización, incluidos los "propios elegibles sujetos de ese patrimonio" (Muriel, 2010). Visto así, la cuestión de la identidad individual y colectiva se relaciona con lo definido como "propio", que en definitiva constituye un patrimonio que define a una comunidad, grupo o colectivo. Los mecanismos o referentes activados por los agentes aportan con una cultura de la memoria local que se manifiesta en los procesos patrimonializadores contemporáneos, revelando en términos macro una política de la memoria.

En el marco de una cultura memorística y nostálgica, el "creciente entusiasmo por la recuperación del pasado nacional" (Samuel, 1996) vuelve imprescindible el determinar los agentes que participan de manera activa en este proceso de preservación/olvido, desde donde una cartografía bibliométrica aporta cuantiosa información de valor para conocer, definir y analizar dichos agentes.

La discusión sobre la construcción de las identidades personales y colectivas es una problematización que para algunos surge en momentos de crisis o cuando hay una autoconciencia o percepción de estar en crisis (Larraín, 2001). Visto así, la pregunta por la identidad de un territorio se instala en una encrucijada o punto de encuentro tensionado dado que en torno a la identidad concurre la interpelación sobre el sentido general construido en la sociedad contemporánea (debate modernidad tardía o postmodernidad y el desafío sobre los relatos totalizantes y esencialistas) y el creciente interés por la construcción de relatos y constitución de prácticas de nuevos actores sociales, relacionadas con la pluralidad de discursos, y por extensión el debate sobre la conservación de elementos (tanto materiales, tangibles como simbólicos e intangibles) en riesgo de desaparecer (debate cultura de la memoria y patrimonio cultural).

\section{Antecedentes}

La cuestión de la identidad del territorio de Playa Ancha a partir del reconocimiento y descripción de la cultura de la memoria emblemática y bibliográfica expone un escenario de disputas en el plano de lo simbólico derivadas de la confrontación de proyectos culturales, socio-económicos y políticos conducidos por diferentes agentes culturales.

El interés por examinar las características de la cultura de la memoria emblemática sobre Playa Ancha radica es que ésta termina por legitimar las acciones patrimonializadoras de ciertos agentes sociales por sobre otros, excluyendo la experiencia y el recuerdo personal que no tiene asidero en el recuerdo colectivamente significativo, revelando las inclinaciones y tendencias de una política de la memoria y por cierto del olvido. La política de la memoria se instituye desde el proceso confrontación y lucha por construir memorias emblemáticas (Stern, 2000), y ocupa un lugar central en la activación de los proyectos de cambio y/o innovación social en las sociedades marcadas por la desigualdad, al considerar que el ethos que se intala determina los eventos y sus relatos recordables bajo la exclusión de otras narrativas (Gascón, 2010). Lo "recordable" de una política de la memoria construida desde la hegemonía fortalece imaginarios aproblemáticos, armónicos, no controvertidos que terminan por desconocer o negar la diversidad de un territorio.

La operación cognitiva, emotiva y política de visitar el pasado, recordar eventos y acontecimientos desde el lugar que proporciona el presente permite abordar las cristalizaciones históricas e hitos constituyentes de la proyección de una sociedad o comunidad hacia el futuro asumiendo que dentro de la heterogeneidad de las experiencias, lo que se tiene en común como colectivo es nuestro pasado (Garretón, 2003). Para los efectos de esta investigación lo que se examina es una estructura de larga duración como matriz de construcción de una memoria emblemática (Bourdieu, 1988) que se remonta al inicio del siglo XX y que establece una relación con la estructura social territorial, las matrices de pensamiento dominantes y las relaciones que definen los intercambios materiales y simbólicos entre los agentes culturales dominantes con mecanismos de reproducción de la memoria institucionalizados, jerárquicos y excluyentes. 


\title{
3 Objetivos y metología aplicada
}

La estretegia de búsqueda de esta investigación abarca desde el año 1900 a 2015. Para recabar la información se han utilizado las bases de datos E-lis, EBSCOhost, Scielo, Cybertesis de la Universidad de Chile, Catálogo de la Biblioteca Nacional de Chile y catálogos de 40 Universidades Chilenas. Para la recuperación de la información se utilizó lenguaje natural así como lenguaje controlado a través de Tesauros y Listados de Encabezamientos de materia.

El número de resultados pertinentes para realizar el análisis alcanza los 254 ítemes y los autores considerados son tanto autores naturales como autores institucionales.

Los objetivos de este estudio son:

- Reconocer los principales discursos bibliográficos del territorio de Playa Ancha

- Explorar nuevos formatos para representar la información bibliográfica, relacionados con la cartografía.

Las etapas que cursó son las siguientes:

1. Diseño de planilla para parametrización de datos bibliográficos

2. Levantamiento documental. Se consultó la bibliografía registrada en los OPAC's de universidades del país y de la región, considerando todas las tipologías documentales existentes. También se consultó la base de datos Scielo, Google Acadmémico y Cybertesis.

3. Poblamiento de los registros en la base de datos con la bibliografía referida al territorio de Playa Ancha.

4. Parametrización de la información recabada.

5. Representación de la información organizada a través de estadísticas y mapas.

6. Análisis y conclusiones de los fenómenos observados.

\section{Discusión de los resultados}

El levantamiento de la información se organizó bajo los siguientes criterios:

- Tipología documental publicada en torno al territorio, dividida en: artículos, tesis, libros y periódicos.

- Materias que se han investigado y que han aportado en la consolidación de la memoria del territorio, dividida en áreas principales o descriptor único, y en descriptores específicos.

- Caracterización de las principales entidades desde donde emergen los discursos que aportan en la cristalización de la memoria emblemática del territorio, entendiendo que para ello existen básicamente autores individuales y corporativos, debidamentficados identificados en el análisis.

- Décadas en las que se producen los documentos.

- Representación geográfica de donde se sitúan las investigaciones realizadas.

El levantamiento bibliográfico indica que las Tesis de Grado constituyen el tipo de documento que más recurrentemente se ha ocupado de estudiar el territorio, entendiendo que las instituciones educativas desempeñan un rol protagónico en la generación de nuevo conocimiento. De este modo, el espacio inmediato se torna el lugar natural a investigar e intervenir por parte de las universidades de la localidad.

En detalle, las temáticas más investigadas son en el siguiente orden decreciente:

\author{
Educación (representa un $38 \%$ ) \\ Bibliografía relacionada con Valores, Educación pre-escolar, Arte y Literatura, \\ Inclusión, Organizaciones sociales, Tecnología y Redes. \\ Desarrollo (representa un 23\%) \\ Bibliografía relacionada con desarrollo Urbano Social, Sistema penitenciario, \\ Desarrollo Económico, Desarrollo Turístico, Ingeniería.
}

Historia (representa un 18\%)

Bibliografía relacionada con Memoria y Patrimonio, historia del Desarrollo Social, Identidad y mitos; y Geografía. 
Arquitectura (representa un 13\%)

Bibliografía relacionada con Patrimonio, Contrucciones religiosas, Construcciones deportivas, Construcciones educacionales, Construcción y segregación

Salud (representa un $\mathbf{8 \%}$ )

Bibliografía relacionada con Embarazo y primera infancia, Calidad de vida, Enfermedades y Medioambiente.

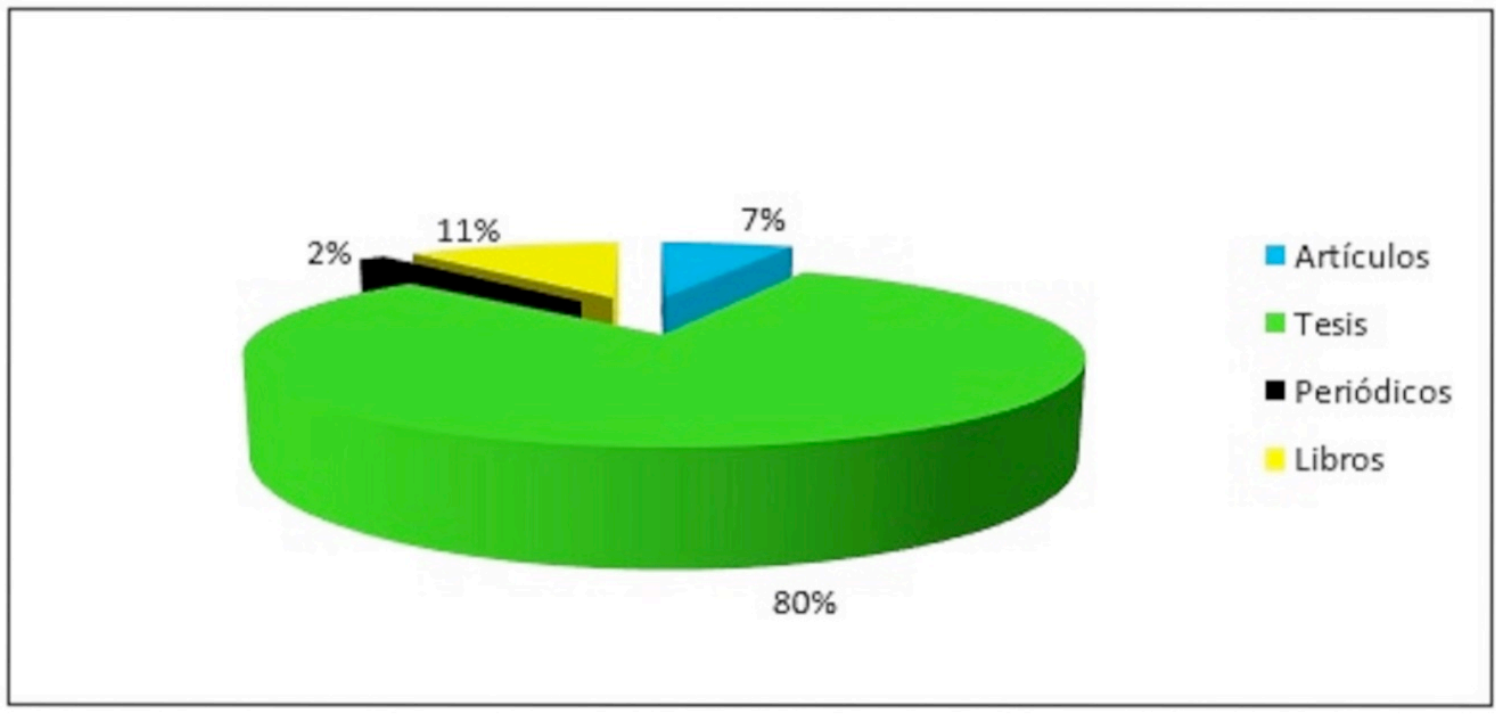

|Gráfico 1 Publicaciones por tipología documental

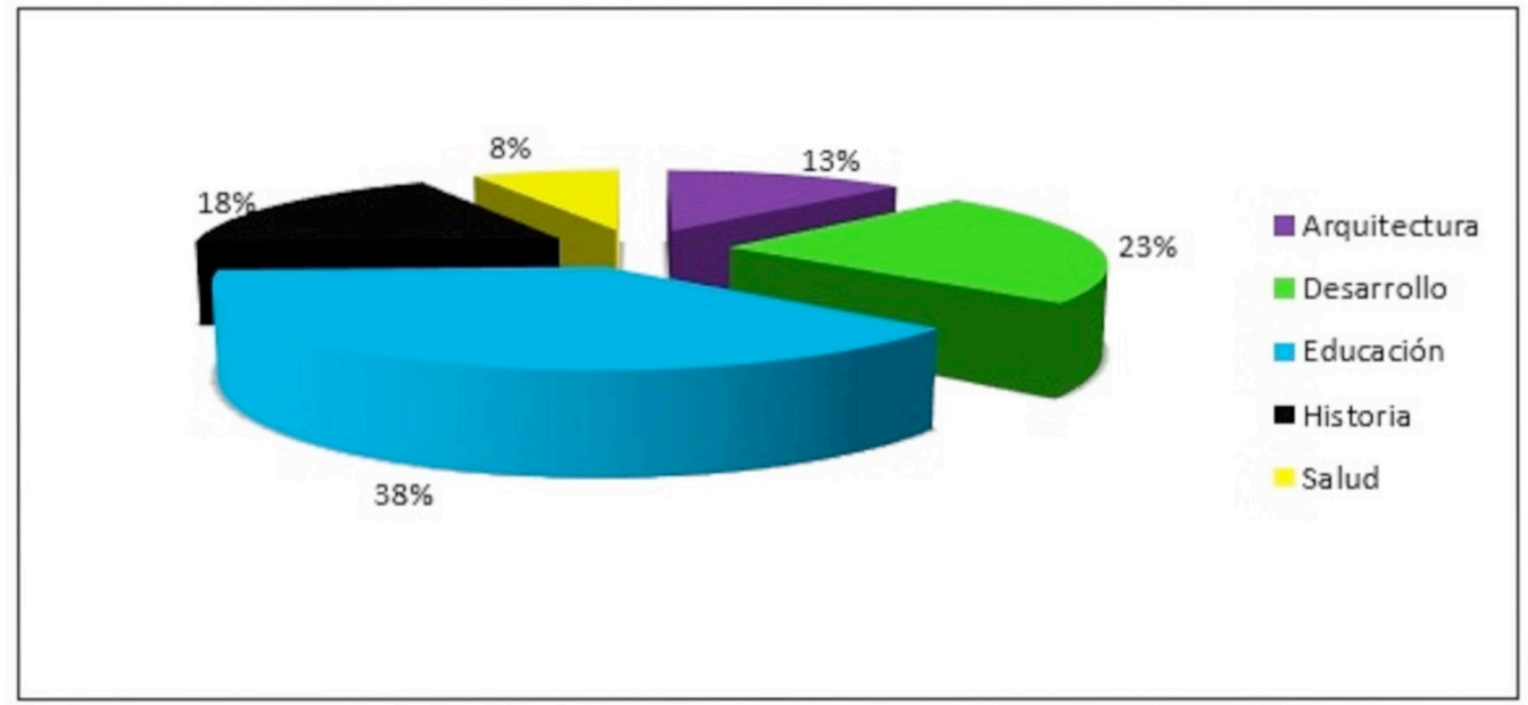

\section{Gráfico 2 Publicaciones por descriptor principal}

Las temáticas de las publicaciones de las Tesis de Grado se centran principalmente en temas de Arquitectura: mientras que en libros y artículos indexados, tratan temáticas relacionados con Historia y con Educación.

También se observa que los temas que preocupan a la comunidad en general no son los mismos que aquellos que ocupan a la institucionalidad educacional, entendiendo que para ésta última lo más intersante de investigar está relacionado con la Arquitectura, especialmente temas patrimoniales y monumentales. Mientras que para los habitantes, para el escritor que se ubica fuera de esta institucionalidad está mucho más interesado en referirse a temas relacionados con Historia, referidos especialmente a memoria local. 
Las Cartografías bibliográficas muestran cómo las universidades de Playa Ancha y de Valparaíso han centrado sus investigaciones en su entorno inmediato, teniendo como espacio de estudio principalmente: Cementerio número 3 Playa Ancha y Deportivo Playa Ancha.

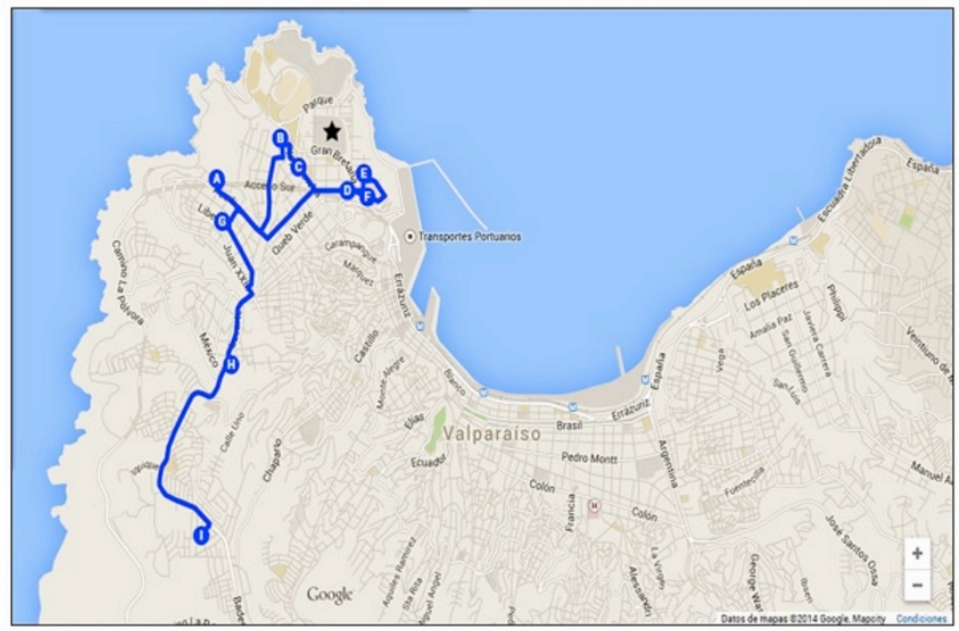

\begin{tabular}{|l|l|}
\hline \multicolumn{2}{|c|}{\begin{tabular}{c}
\multicolumn{1}{|c|}{ AGENTE EXPERTO } \\
UNIVERSIDAD DE VALPARAÍso
\end{tabular}} \\
\hline A & Cementerio No3 \\
\hline B & Escuela Diego Portales \\
\hline C & Deportivo Playa Ancha \\
\hline D & Teatro Odeón \\
\hline E & Vivienda, Santa María 251 - 253 \\
\hline F & $\begin{array}{l}\text { Vivienda, Calle Pedro León Gallo } \\
\text { 413 }\end{array}$ \\
\hline G & Colegio Reino de Suecia \\
\hline H & Parroquia Marina Mercante \\
\hline I & Escuela República Árabe - Siria \\
\hline
\end{tabular}

Cartografia bibliográfica 1

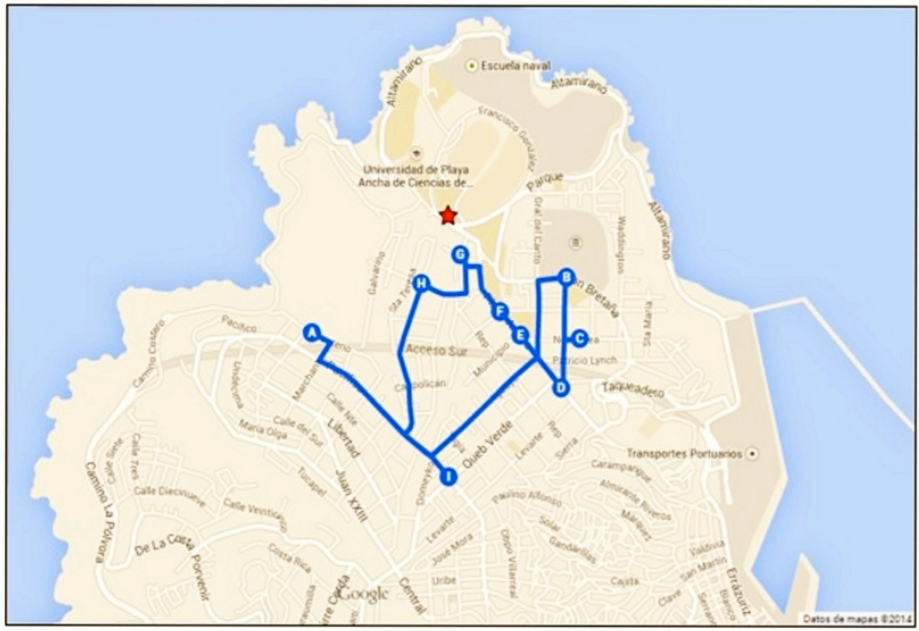

\begin{tabular}{|l|l|}
\hline \multicolumn{2}{|c|}{$\begin{array}{l}\text { AGENTE EXPERTO } \\
\text { UNIVERSIDAD DE PLAYA ANCHA }\end{array}$} \\
\hline A & Cementerio N ${ }^{\circ}$ \\
\hline B & Biblioteca Liceo de niñas de Playa Ancha \\
\hline C & $\begin{array}{l}\text { Capilla Nuestra Señora del Rosario, } \\
\text { Convento de los Dominicos }\end{array}$ \\
\hline D & Biblioteca Municipal Rotaria \\
\hline E & Liceo Politécnico Alfredo Nazar Feres \\
\hline F & Deportivo Playa Ancha \\
\hline G & Escuela Diego Portales \\
\hline H & $\begin{array}{l}\text { Centro de Tránsito y Distribución de } \\
\text { Playa Ancha }\end{array}$ \\
\hline I & Consultorio Quebrada Verde \\
\hline
\end{tabular}

Cartografia bibliográfica 2

\section{Conlusiones}

Los estudios bibliométricos representan cuestiones que van más allá de los meros índices cuantitativos de publicaciones y temáticas, la mayor parte de las veces vinculados a la generación de capital a través del conocimiento científico. En el caso de la cartografía bibliográfica, resulta interesante comprender de qué manera esta representación muestra una lógica social hasta ahora invisibilizada. En el caso de este estudio, es posible observar cómo las universidades de una localidad determinada han ido dictando las temáticas que interesan y preocupan a un cierto grupo que perteneciendo al territorio, ha ido construyendo a partir de estudios, tesis de grados y artículos en general, una huella bibliográfica, que ha ido cristalizando la memoria de la localidad. A su vez, este discurso oficial, hegemónico, autocrático se ha propcupado principalmente de su territorio más inmediato, invisibilizando los sectores más marginados y marginales de la localidad: los espacios sociales alejados de estos centros educacionales no están en la agenda de los temas a estudiar y por lo tanto prácticamente no existen publicaciones en temáticas relacionadas a sectores como Puertas Negras o Montedónico, que son justamente los sectores más vulnerables de Playa Ancha. Dentro de este discurso, poco o nada han tenido por decir los mismos habitantes de este territorio, puesto que si bien existen publicaciones independientes, son escasas respecto a las publicaciones provenientes del mundo académico. 
Los estudios métricos a pequeña escala se vuelven una herramienta interesante en cuanto permiten conocer los sesgos de los mega-estudios. La idea de explorar cartografías de información centradas en determinados espacios geográficos, es justamente la de incorporar otros elementos a la hora de analizar la información.

La cartografía bibliométrica, permite mover el eje central de los estudios, comprendiendo que ellos constituyen fragmentos de la realidad, instalando una discusión que plantea un asunto ético, de inclusión y de descentralización: toda propuesta es una opción de construcción de la cultura, de la realidad y de la sociedad.

Es necesario leer críticamente las prácticas dominantes en la formas de conocimiento que se han instaurado en los estudios bibliométricos, practicando otras formas de escala, proyección y simbolización de la información como prácticas democratizadoras, tal como lo propone una cartografía bibliográfica, en dirección de recuperar el vínculo entre el la producción bibliográfica y el territorio (Mardones, 2015).

Es necesario reconfigurar la lógica del conocimiento científico y posibilitar la creación de formas innovadoras de intervención cultural y política de carácter democrático y emancipatorio: "Este objetivo exige una nueva configuración de los saberes y de ecologías del conocimiento. Es decir, una revisión crítica de las jerarquías y de las fronteras culturales que naturalizan y presentan como inmutables la separación entre ciencias y humanidades, ciencias sociales y ciencias sociales, naturaleza y cultura, expertos e ignorantes, conocimiento especializado y sentido común" (Rodríguez, 2009).

\section{Referencias}

Ariño, A. (2015). La patrimonialización de la cultura y sus paradojas postmodernas.

http://www.academia.edu/1198690/La patrimonializacio n de la cultura y sus paradojaspostmodernas (Recuperado 26 de junio de 2015)

Bourdieu, P. (1988). La distintición: criterio y bases sociales del gusto. Madrid: Taurus.

Di Pietro, L. (2011). Hacia un desarrollo integrador y equitativo: una introducción al desarrollo local. http://www. bibliotecavirtual.info/2011/06/hacia-un- desarrollo-integrador-y- equitativo-una-introduccion-al-desarrollo-local/ (Recuperado 02 de junio de 2015)

Garretón, M. (2003). Memoria y proyecto de país. Revista de Ciencia Política Santiago, vol. 23 (2), 215-230.

http://doi.org/10.4067/S0718-090X2003000200010 (Recuperado 26 de junio de 2015)

Gascón, F. (2010). ¿Políticas de la memoria o semióticas del olvido? Ima(r)ginarios sobre comunicación y cambio social. Razón y Palabra 2010, vol 15, (71), 1-13 http://www.redalyc.org/articulo.oa?id=199514914018 (Recuperado 26 de junio de 2015)

Larraín, J. (2014). La identidad chilena. Chile; LOM, 250 p.

Mardones, M. (2015). La cartografía simbólica y las representaciones bibliométricas.

Márquez, F. (2003). Identidad y fronteras urbanas en Santiago de Chile. Psicología Em Revista, vol. 10 (14), 35-51.

Muriel, D. (2010). Estados hipermnésicos en la cultura de la memoria y la inflación patrimonializadora: algunos apuntes en torno a la labor experta sobre la memoria de la Guerra Civil Española. Papeles del CEIC 62, vol 2 (64), 1-38. http://www.redalyc.org/articulo.oa?id=76517019002 (Recuperado 26 de junio de 2015)

Rodríguez, J. (2009). Los usos sociales de la ciencia: tecnologías convergentes y democratización del conocimiento. (Spanish). The Social Uses of Science: Converging Technologies and the Democratization of Knowledge. (English), 17(34), 225-249.

Samuel, R. 1996. Theatres of Memory: Past and Present in Contemporary Culture. Londres; Verso, 320 p.

Sousa-Santos, B. (1991). Una cartografía simbólica de las representaciones sociales: prolegómenos a una concepción posmoderna del Derecho. Venezuela; Nueva Sociedad vol. 116, 18-38.

Stern, S. (1998). De la memoria suela a la memoria emblemática: Hacia el recordar y I olvidar como procesos histórico (Chile, 1973 - 1998). Uruguay; Biblioteca Virtual. http://www.cholonautas.edu.pe/modulo/upload/SStern.pdf (Recuperado 26 de junio de 2015) 


\section{Datos de los autores}

Marjorie Mardones Leiva

Bibliotecóloga, Licenciada en Ciencias de la Documentación y Magister en Arte con mención en Patrimonio por Universidad de Playa Ancha (CHILE), con estudios de Licenciatura en Castellano por la Universidad de Concepción (CHILE). Actualmente se desempeña como Académica Investigadora del Departamento de Ciencias de la Documentación de la Facultad de Ciencias Sociales de la Universidad de Playa Ancha (CHILE). Se ha destacado por su activa participación en el ámbito cultural con diversas publicaciones en los ámbitos artístico, empresarial y académico. Integra el Centro de estudios del Patrimonio Oceánico de la Universidad de Valparaíso (CHILE), CIDEO e integra el Observatorio de Participación social y Territorio de la Universidad de Playa Ancha. Se ha dedicado a estudiar la historia del libro y las prácticas de lectura, el patrimonio documental y el libro como dispositivo de la memoria social.

marjorie.mardones@upla.cl

\section{Javiera Carmona Jiménez}

Periodista, Magister en Arqueología y cursó el Doctorado en Historia (Mención Etnohistoria), ambos en la Universidad de Chile. Es académica e investigadora de la Universidad de Playa (Valparaíso) en estudios de pregrado y postgrado (Magister en Arte - Mención Patrimonio y Magister en Educación Artística) sobre cultura y comunicación, y su línea de investigación es de corte interdisciplinario, en las que se cruza la historiografía, comunicación y estética. Entre sus últimos trabajos cabe mencionar la relación de los museos chilenos con sus audiencias, memoria de la catástrofe y fotografía en Chile, estudio comparado de la prensa regional en Chile sobre el tratamiento del movimiento estudiantil del 2011 y la historia de la arqueología chilena a partir de la trayectoria de Hans Niemeyer Fernández. También ha integrado el jurado del concurso del Fondo Nacional del Libro y la Lectura (Consejo de la Cultura y las Artes de Chile).

javiera.carmona@upla.cl

Recibido - Received: 2016-06-01

Aceptado - Accepted: 2016-09-14

\section{(cc) BY}

This work is licensed under a Creative Commons Attribution 4.0

United States License.

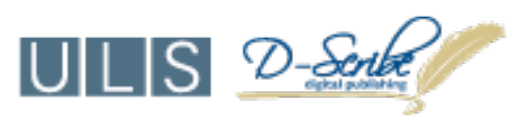

This journal is published by the University Library System of the University of Pittsburgh as part of its

D-Scribe Digital Publishing Program and is cosponsored by the University of Pittsburgh Press. 